\title{
Continuous exposure of PM2.5 exacerbates ovalbumin-induced asthma in mouse lung via a JAK-STAT6 signaling pathway
}

\author{
Yonghui Yang ${ }^{1, A-C}$, Xiaoxia Li ${ }^{1, B, C}$, Xiaoying An ${ }^{2, B-D}$, Ling Zhang ${ }^{3, B, C}$, Xingbin Li3 ${ }^{3, B C}$, , Liang Wang ${ }^{3, B, C}$, Guiyun Zhu ${ }^{1, D-F}$ \\ ${ }^{1}$ Department of Pathology, Hebei Province Chest Hospital, Lung Cancer Prevention and Control Center of Hebei Province, Shijiazhuang, China \\ ${ }^{2}$ Laboratory of Molecular Biology, Hebei Province Chest Hospital, Lung Cancer Prevention and Control Center of Hebei Province, Shijiazhuang, China \\ ${ }^{3}$ Department of Pneumology, Hebei Province Chest Hospital, Lung Cancer Prevention and Control Center of Hebei Province, Shijiazhuang, China \\ A - research concept and design; $\mathrm{B}$ - collection and/or assembly of data; $\mathrm{C}$ - data analysis and interpretation; \\ $\mathrm{D}$ - writing the article; $\mathrm{E}$ - critical revision of the article; $\mathrm{F}$ - final approval of the article
}

\section{Address for correspondence}

Guiyun Zhu

E-mail:zgyblk@163.com

Funding sources

The China government funds of excellent clinical medical talents and basic research projects.

Conflict of interest

None declared

Received on September 1, 2019

Reviewed on December 28, 2019

Accepted on April 24, 2020

Published online on June 28, 2020

\begin{abstract}
Background. Epidemiological studies and mice models have demonstrated that air pollution containing particulate matter smaller than 2.5 um (PM2.5) exacerbates acute episodes of asthma in both children and adults.

Objectives. To investigate the effect of continuous PM2.5 treatment on asthma regulation mechanism behind this effect.

Material and methods. In this study, the effects of continuous exposure to PM2.5 on asthma and eosinophil recruitment was compared to the effect of a single pre-ovalbumin (OVA)-sensitization exposure to PM2.5. Wild-type mice were either challenged once with PM2.5 + OVA before sensitization and asthma induction over a 27-day period, or with 5 times of PM2.5 + OVA treatment and sensitization/asthma induction over the same period.

Results. Continuous exposure to PM2.5 significantly increased total plasma immunoglobulin E (lgE), bronchial alveolar lavage fluid (BALF) cell numbers, eosinophils, and macrophages, leading to increased lung injury. This effect was regulated through increased production of chemokines and cytokines, such as interleukin (IL)-1B, monocyte chemoattractant protein 1 (MCP-1), IL-12, LL-5, LL-13, and prostaglandin D2 (PGD 2). Eosinophil recruitment during continuous PM2.5 treatment was regulated through phosphorylation of the JAK/STAT6 pathway. As this study shows, continuous PM2.5 treatment significantly worsens asthma as compared to single exposure to PM2.5 or OVA exposure alone.
\end{abstract}

Conclusions. Our findings reveal that continuous exposure of PM2.5 exacerbates OVA-induced asthma in mouse lung through JAK-STAT6 signaling pathway.

Key words: inflammation, asthma, PM2.5, JAK-STAT6 pathway

Cite as

Yang Y, Li X, An X, et al. Continuous exposure of PM2.5 exacerbates ovalbumin-induced asthma in mouse lung via a JAK-STAT6 signaling pathway. Adv Clin Exp Med. 2020;29(7):825-832. doi:10.17219/acem/121009

DOI

10.17219/acem/121009

Copyright

Copyright by Author(s)

This is an article distributed under the terms of the

Creative Commons Attribution 3.0 Unported (CC BY 3.0)

(https://creativecommons.org/licenses/by/3.0/) 


\section{Introduction}

A combination of enormous population growth, rapid urbanization and industrialization in China has led to a massive increase of air pollution, a phenomenon previously observed in developed countries. ${ }^{1-3}$ A detrimental effect from increased air pollution is that the general population is exposed to harmful air pollutants containing particulate matter (PM). Particulate matter contains an extensive variety of toxic substances derived from industrial and vehicular emissions, coal combustions, sea salt, and soil dusts. ${ }^{3}$ Depending on the city where the pollutant is collected, PM can contain varying amounts of fossil fuel combustion remains, such as polycyclic aromatic hydrocarbons, sulfates $\left(\mathrm{SO}_{4}{ }^{2-}\right)$, nitrates $\left(\mathrm{NO}^{-}\right)$, microbial material, and chemical elements such as iron, zinc, silica, sodium, and aluminium. ${ }^{4-6}$ Furthermore, these PMs can remain in the atmosphere for a lengthy amount of time, transverse great distances and easily trigger lung disease. ${ }^{7}$ It has been shown that the smaller the particle size, the greater injury PM causes to the lungs. ${ }^{8}$

Particulate matter has been categorized into 2 grades: PM10, whose aerodynamic diameter is less than or equal to $10 \mu \mathrm{m}$, and PM2.5, whose aerodynamic diameter is less than or equal to $2.5 \mu \mathrm{m}$. PM2.5, a fine particulate matter, poses the greatest risk, as it induces stronger pulmonary and allergic inflammatory responses as compared to larger counterparts when tested at an equal mass dose. ${ }^{9,10}$ This was mainly due to a larger surface area to mass ratio. It must also be noted that, in addition to PM surface area, the chemical composition is also an important determinant of the inflammatory response. Since Asian dust aerosols can spread over a large area, including East Asia, the Korean peninsula and Japan, during spring, anthropogenic pollution has become a major public health concern in Eastern Asia. ${ }^{11}$

Asthma, in both children and adults, is a common respiratory disease worldwide. Asthma is characterized by chronic allergic airway inflammation, with episodes of superimposed acute inflammation. ${ }^{12}$ Studies have now shown that PM2.5 is correlated with the exacerbation of asthma in Europe, the Americas, Korea, Japan, and China, ${ }^{13-17}$ where ambient air PM2.5 increases such incidences as asthma-related emergency department visits, worsened wheezing and dyspnea, and children hospital admissions for asthma. ${ }^{18-21}$ Asthma morbidity is also positively associated with daily ambient PM2.5 levels. In Asia, daily admissions in the clinic for asthma, acute respiratory events in children as well as allergic rhinitis in Taipei, Taiwan coincided with Asian dust storm events containing toxic pollutants. ${ }^{22-25}$

During acute exacerbations of asthma, clinical manifestations include eosinophilic airway inflammation in the distal portion of the lung with symptom exaggeration, such as cough, chest tightness and dyspnea. ${ }^{26}$ During presentation of asthma, the typical cytokine profile is generally observed as a $\mathrm{T}_{\mathrm{H}} 2$-type airway inflammation with increased levels of eosinophils, immunoglobulin $\mathrm{E}$ (IgE) and the production of $\mathrm{T}_{\mathrm{H}} 2$-related cytokines such as interleukin (IL)-4, IL-5 and IL-13. ${ }^{27,28}$ Interleukin 4 is necessary for the polarization of $\mathrm{T}$ helper cells towards a $\mathrm{T}_{\mathrm{H}}$ 2-phenotype, ${ }^{29} \mathrm{IL}-5$ helps promote proliferation and differentiation of $\mathrm{B}$ cells $\mathrm{s}^{30}$ and IL-13 induces the induction of $\mathrm{T}_{\mathrm{H}} 2$ cells. ${ }^{31}$ Apart from eosinophil recruitment, macrophages are described to play a role in inflammation during allergic airway inflammation. ${ }^{32,33}$ In addition, the TLR2/ TLR4/MyD88-signaling pathway has been shown to regulate the exacerbation of murine lung eosinophilia during PM2.5/ovalbumin (OVA)-induced asthma. ${ }^{34}$

In this study, urban PM was harvested from the atmosphere in Hebei, China. PM2.5 was administered to mice, together with OVA, to examine the effects of long-term exposure of pollutants on asthma exacerbation. This effect was compared to a short-term sensitization exposure of PM2.5 to mice. The study investigated pathological changes of lungs, cellular infiltration in the bronchoalveolar lavage fluid (BALF), inflammatory cytokine changes in BALF, OVA-specific IgE antibodies in serum, as well as the role of the JAK/STAT signaling pathway in the recruitment of eosinophils into the lungs. This study was performed to determine the inflammatory mechanism responsible for asthma exacerbation resulting from longterm exposure to PM2.5 pollutants.

\section{Material and methods}

\section{Experimental animals}

$\mathrm{BALB} / \mathrm{c}$ mice were kept in pathogen-free conditions and received sterile OVA-free food and water. The mice were maintained on a 12-hour light/dark cycle, under constant room temperature $\left(24^{\circ} \mathrm{C}\right)$ and relative humidity $(40-80 \%)$. The experimental protocols shown in this study were approved by the Institutional Animal Care and Research Advisory Committee at Chongqing Medical University, China. The use of animals in these experiments was in accordance with the guidelines issued by the Chinese Council on Animal Care.

\section{PM2.5 particles preparation}

Methods of preparing PM2.5 were provided by the $\mathrm{Hu}-$ bei Province Center for Disease Control and Prevention, China. First, PM2.5 was collected on a glass fiber filter using a Thermon Anderson high-volume sampler (Thermon Anderson, San Marcos, USA). The sample membrane was equilibrated at $60^{\circ} \mathrm{C}$ for $6 \mathrm{~h}$ before weighing using an analytical balance. The ambient PM2.5 concentration was calculated from sample weight and air volume. PM2.5 was recovered from the membrane using a modification of an aqueous extraction technique: the PM2.5-adsorbed 
membrane was cut into $1 \times 3 \mathrm{~cm}^{2}$ size and immersed in ultrapure water for eluting PM2.5, then 'cold-shocked' for $30 \mathrm{~min}$ at low temperature 3 times. Finally, PM2.5 was eluted with gauze filtration, vacuum freeze-dried and stored at $-20^{\circ} \mathrm{C}$ for future use or sale.

\section{Study protocol}

Four groups ( $\mathrm{n}=10$ per group) of WT mice were prepared for treatment with particles as shown in Fig. 1: (A) control group ( $\mathrm{n}=10)$; (B) OVA group $(\mathrm{n}=10)$; (C) OVA + PM2.5 pre-treated group; and (D) OVA + PM2.5 long-treatment group. For OVA-induced mouse model of asthma, mice were immunized intraperitoneally with $50 \mathrm{mg}$ of OVA in $1 \mathrm{mg}$ of $\mathrm{Al}(\mathrm{OH})_{3}$ in $200 \mathrm{~mL}$ saline on days 0,7 and 14. On days 21, 22 and 23, the mice were nebulized with $300 \mathrm{mg}$ of OVA in $5 \mathrm{~mL}$ saline for $25 \mathrm{~min}$ per day. For PM2.5 treatment, the mice were treated with $100 \mu \mathrm{g}$ in sterile phosphate-buffered saline (PBS; $50 \mathrm{~mL}$ final volume), where PM2.5 was slowly instilled, followed with $200 \mathrm{~mL}$ of clean air. For PM2.5 pre-treatment, the mice were treated with PM2.5 for 7 days from day 0 to day 7. For PM2.5 long-treatment, the mice were treated with PM2.5 for 27 days from day 0 to day 27 .

\section{Lung tissue histology analysis}

Lung tissues from the mice were fixed with $10 \%$ formalin in saline and embedded in paraffin. The paraffin blocks were serially sectioned at $5-\mu \mathrm{m}$ thickness and stained with hematoxylin-eosin (H\&E) staining for lung histology analysis.

\section{BALF analysis}

Within $24 \mathrm{~h}$ after the final challenge, the mice were sacrificed, and the trachea was cannulated. Plasma and BALF were collected from mice at harvest. The BALF was obtained by flushing the lungs 3 times with $1.5 \mathrm{~mL}$ of PBS. The BALF cellular typing was determined with cytospin preparation and Wright-Giemsa staining, followed by counting total cell number, macrophages and eosinophils. Plasma IgE (Invitrogen, Carlsbad, USA), BALF IL-1 $\beta$ (eBioscience, Waltham, USA), MCP-1 (eBioscience), IL-12 (eBioscience), IL-5 (eBioscience), IL-13 (PeproTech, Stockholm, Sweden), and prostaglandin D2 (PGD 2 ; Caymanchem, Ann Arbor, USA) were determined using enzyme-linked immunosorbent assay (ELISA) kits according to the manufacturers' instructions.

\section{Western blot}

The total proteins from mice lung tissues were isolated, and the protein concentrations were determined using a bicinchoninic acid assay. The proteins from whole-cell lysates were used for western blot. Anti-JAK1, anti-p-JAK1,
anti-JAK2, anti-p-JAK2, anti-STAT6, anti-p-STAT6, and GAPDH (all purchased from Cell Signaling Technology, Leiden, the Netherlands) antibodies were used. Densitometry of bands from western blots was measured using Image J software (National Institutes of Health, Bethesda, USA), and the density of the PJAK2 or p-STAT6 proteins relative to total JAK or total STAT6 was calculated.

\section{Cell culture}

Bone marrow-derived eosinophils were cultured in Iscove's modified Dulbecco's medium (IMDM; Invitrogen) with $20 \% \mathrm{FBS}$ (Invitrogen), $100 \mathrm{IU} / \mathrm{mL}$ penicillin and $10 \mathrm{mg} / \mathrm{mL}$ streptomycin, $2 \mathrm{mM}$ glutamine, $25 \mathrm{mM}$ HEPES, $1 \times$ nonessential amino acids, $1 \mathrm{mM}$ sodium pyruvate and $0.006 \%$ o-mercaptoethanol (Sigma-Aldrich, St. Louis, USA) supplemented with $100 \mathrm{ng} / \mathrm{mL} \mathrm{rmSCF}$ (PeproTech) and $100 \mathrm{ng} / \mathrm{mL}$ recombinant murine FLT-3 ligand (rmFLT3-L; PeproTech) from days 0 to 4 . On day 4, the medium was replaced with medium containing $10 \mathrm{ng} / \mathrm{mL}$ rmIL-5 (PeproTech). The cells were provided with fresh medium supplemented with rmIL -5 every 3 days until to 14 days. A total of $1 \times 10^{6}$ bone marrow-derived eosinophils per group were treated with $5.0 \mu \mathrm{g} / \mathrm{cm}^{2} \mathrm{PM} 2.5$ for $48 \mathrm{~h}$ with or without $100 \mathrm{ng} / \mathrm{mL}$ ruxolitinib (InvivoGen, San Diego, USA). After $48 \mathrm{~h}$, the cell supernatants were harvested. The concentrations of IL-1 $\beta$, IL-5 and IL-13 in the supernatants were determined using ELISA.

\section{Statistical analyses}

The data was expressed as the mean and standard error of the mean (SEM). All data was analyzed using SPSS v. 17.0 software (SPSS Inc., Chicago, USA). Comparisons among experimental groups were performed with non-parametric Kruskal-Wallis test (one-way analysis of variance (ANOVA) on ranks) due to multiple group comparisons and abnormal data distribution. Differences with p-values less than 0.05 were considered to be statistically significant.

\section{Results}

\section{Pathological changes in airways after PM2.5 pre-treatment and long treatment}

Mice were either pre-treated with PM2.5 twice a day (day 0 and day 7) followed by OVA administration on day 21 (OVA + PM2.5 pre-treated group) or were subjected to a mix of PM2.5 (day 0, 7, 14, 21, and 27) and OVA administration (day 21 to 27) (OVA + PM2.5 long-treated group) (Fig. 1). Ovalbumin treatment with or without PM2.5 treatment induces asthma. ${ }^{35}$ To examine airway changes after OVA and PM2.5 treatment, histopathological analysis on mice lung tissue was conducted. In the control group, mice lung tissue had neatly arranged airway epithelial cells with clear 


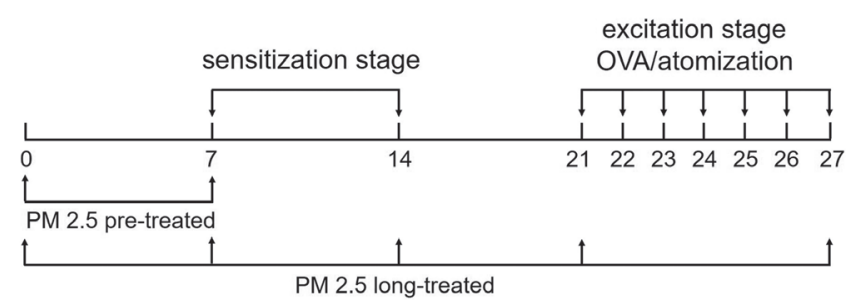

Fig. 1. Diagram outlining treatment stages of mice before OVA-induced asthma. Mice from pre-treated group were treated with PM2.5 (7 days) before sensitization for an additional 7 days. The mice were then subjected to OVA on day 21 to induce asthma. In another treatment group (long-treatment group), mice were treated with PM2.5 every 7 days (on day $0,7,14,21$, and 27). On day 21 to 27 , asthma was induced in mice through OVA treatment

bronchi and alveoli (Fig. 2, control group). Ovalbumin treatment alone resulted in the disruption of the ordered lung tissue structure causing widened alveolar septum, capillary dilation and infiltration of leukocytes (eosinophils shown with arrows) (Fig. 2, OVA group). Combining PM2.5 treatment with OVA caused the further breakdown of the lung tissue with increased alveolar septum widening and capillary dilation (Fig. 2, OVA + PM2.5 pre-treated and long-treated group). Furthermore, increased infiltration of leukocytes was observed with both PM2.5 treatment methods with OVA (pre-treatment and long treatment). However, more eosinophils in PM2.5 long treatment with OVA compared to PM2.5 pre-treatment with OVA were observed (Fig. 2, OVA + PM2.5 long-treated group).

\section{PM2.5 treatment induces increased eosinophil infiltration into the lungs}

High levels of IgE are a typical indicator of asthma. Although OVA treatment alone induced increases in plasma IgE compared to the control, both PM2.5 exposures with
OVA (pre-treatment and long treatment) significantly increased plasma IgE levels compared to OVA treatment alone $(\mathrm{p}<0.05$, Fig. 3A).

To analyze cellular infiltration into the lungs in more detail, BALF was used to examine total leukocyte numbers together with eosinophils and macrophages. Ovalbumininduced asthma alone did increase total cell numbers, eosinophils and macrophages as compared to control group ( $<<0.001$, Fig. 3B; $\mathrm{p}<0.001$, Fig. $3 \mathrm{C}$; and $\mathrm{p}<0.01$, Fig. 3D). However, pre-treatment with PM2.5 and OVA significantly increased total cell numbers as well as eosinophil infiltration compared to OVA treatment alone $(\mathrm{p}<0.05$, Fig. 3B; and $\mathrm{p}<0.05$, Fig. $3 \mathrm{C}$ ) but not macrophages. With continuous long treatment of PM2.5 and OVA, total cell numbers, eosinophils and macrophages in the lung were increased as compared to OVA treatment alone $(\mathrm{p}<0.001$, Fig. 3B; $\mathrm{p}<0.001$, Fig. $3 \mathrm{C}$; and $\mathrm{p}<0.05$, Fig. 3D).

\section{Increased inflammatory cytokines after PM2.5 treatment}

Cytokine levels from BALF in control, OVA-treated, and PM2.5 pre- and long-treated mice were examined. The BALF levels of IL-1 $\beta$, MCP-1, IL-12, IL-5, IL-13, and $\mathrm{PGD}_{2}$ were increased after OVA treatment $(\mathrm{p}<0.05$ for IL-1 $\beta$; $\mathrm{p}<0.05$ for MCP-1; $\mathrm{p}<0.01$ for IL-12; $<<0.001$ for IL-5; $\mathrm{p}<0.05$ for IL-13; and $\mathrm{p}<0.05$ for $\mathrm{PGD}_{2}$; Fig. 4A-F). Pre-treatment with PM2.5 followed by OVA increased BALF MCP-1 and IL-5 ( $\mathrm{p}<0.05$ for MCP-1, Fig. 4B; and $\mathrm{p}<0.05$ for IL-5, Fig. 4D) but not IL-1, IL-12, IL-13, and $\mathrm{PGD}_{2}$ (Fig. 4A,C,E,F). Long treatment of PM2.5 with OVA increased IL-1 $\beta$ ( $p<0.05$, Fig. 4A), MCP-1 ( $<<0.05$, Fig. 4B), IL-12 ( $<<0.05$, Fig. 4C), IL-5 ( $<<0.01$, Fig. 4D), IL-13 ( $\mathrm{p}<0.01$, Fig. 4E), and $\mathrm{PGD}_{2}$ ( $\mathrm{p}<0.01$, Fig. 4F) in the BALF.
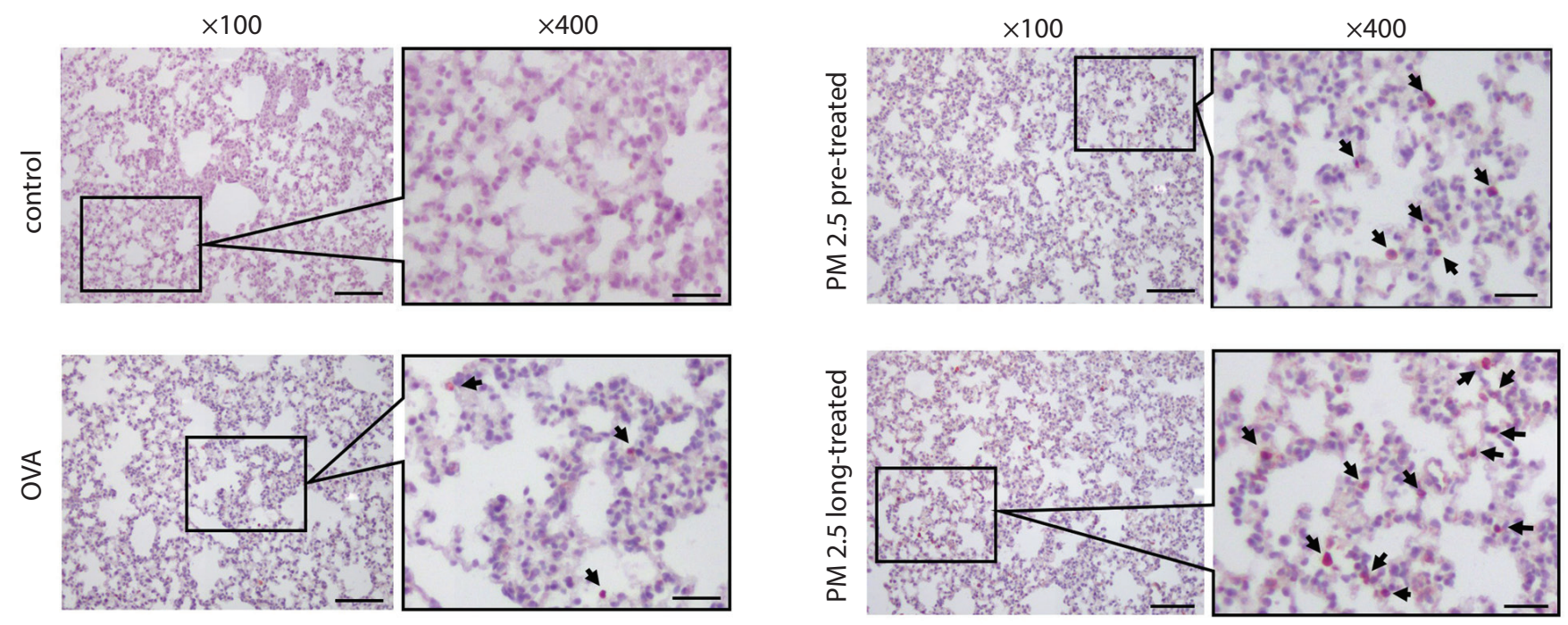

Fig. 2. Comparison of eosinophils in lung tissue after treatment with PM2.5. Magnification $\times 10$ and $\times 40$ of (A) control group, (B) OVA group, (C) OVA + PM2.5 pre-treated group, and (D) OVA + PM2.5 long-treatment group lung samples H\&E-stained for eosinophil infiltration (arrows). Magnification $\times 10$ scale bar: $100 \mu \mathrm{m}$. Magnification $\times 40$ scale bar: $25 \mu \mathrm{m}$ 
A

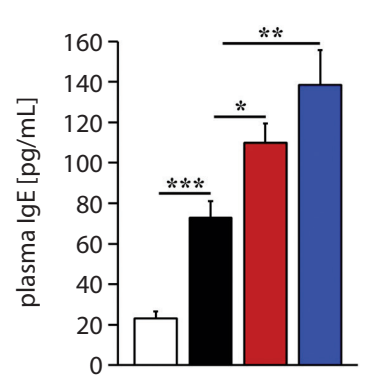

C

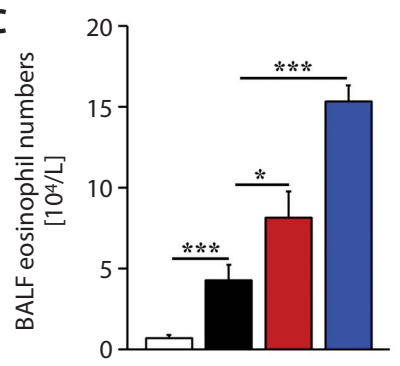

B

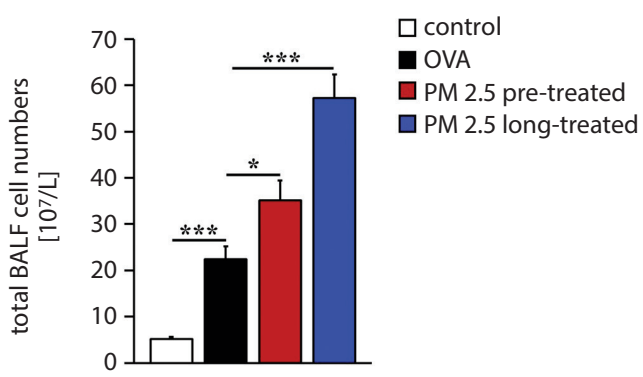

Fig. 3. PM2.5 treatment increases plasma IgE and leukocyte infiltration in lung. Measurement of (A) plasma lgE, (B) total cell numbers from the bronchoalveolar lavage fluid (BALF), (C) total BALF eosinophil numbers, and (D) total BALF macrophage numbers in control group (white bars), OVA group (black bars), OVA + PM2.5 pre-treated group (red bars) and OVA + PM2.5 long-treated group (blue bars)

$n=10, p^{*}<0.05, p^{* *}<0.01, p^{* * *}<0.001$
A

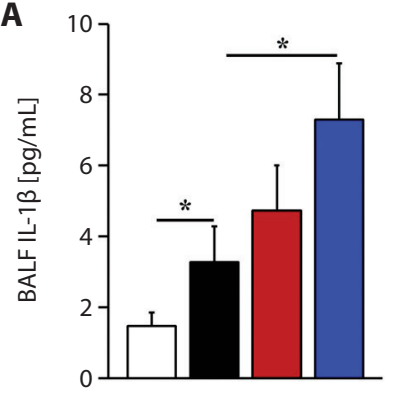

D

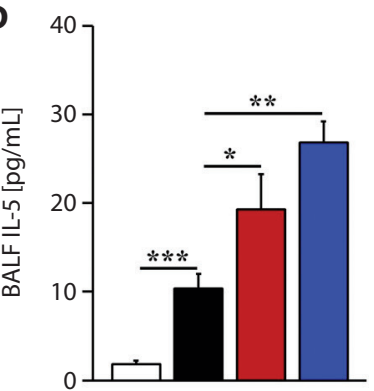

B

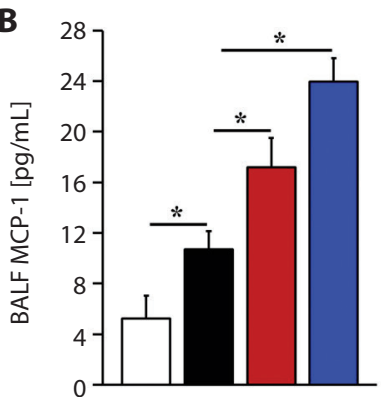

$\mathbf{E}$

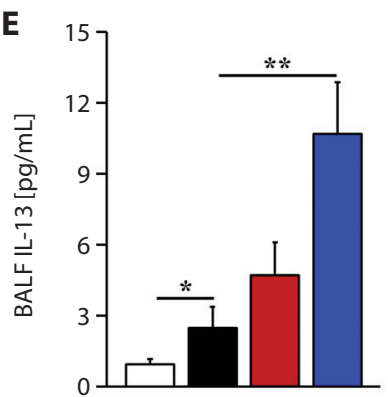

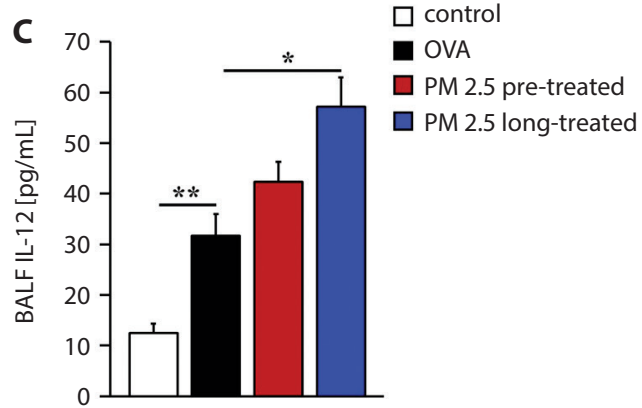

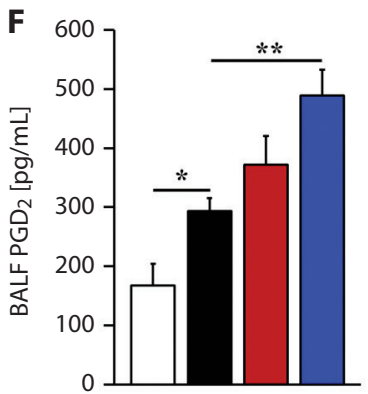

Fig. 4. PM2.5 treatment increases inflammatory cytokine levels in lungs. Measurements of BALF (A) IL-1B, (B) MCP-1, (C) IL-12, (D) IL-5, (E) IL-13, and (F) PGD2 in the control group (white bars), OVA group (black bars), OVA + PM2.5 pre-treated group (red bars), and OVA + PM2.5 long-treatment group (blue bars) $n=10, p^{*}<0.05, p^{* *}<0.01, p^{* *}<0.001$.

\section{JAK-STAT6 pathway regulates eosinophil infiltration and inflammatory cytokine production in lungs}

To determine the signaling pathway that regulated recruitment of eosinophils, ${ }^{36}$ protein levels of non-phosphorylated and phosphorylated JAK/STAT6 were examined with western blotting (Fig. 5A) and quantified using densitometry. Ovalbumin increased the phosphorylation of JAK1, JAK2 and STAT6 compared to control group by about twofold ( $\mathrm{p}<0.05$, Fig. 5B). Long treatment with PM2.5 increased PJAK1 by fourfold compared to the control group and about twofold compared to OVA treatment alone, while pJAK2 was increased by 4-fold compared to the control group and about 1.5-fold compared to OVA treatment alone. Phosphorylation of STAT6 was increased 2-fold after OVA treatment alone while PM2.5 long treatment increased pSTAT6 by 2.5-fold compared to the control group and 1.5-fold compared to OVA treatment alone. No changes in GADPH protein levels were observed (Fig. 5A). Moreover, 
A

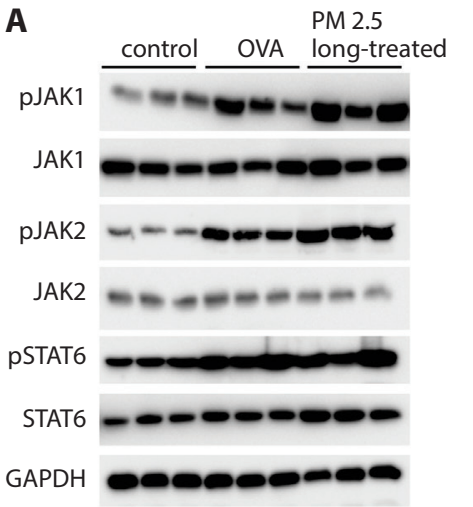

C

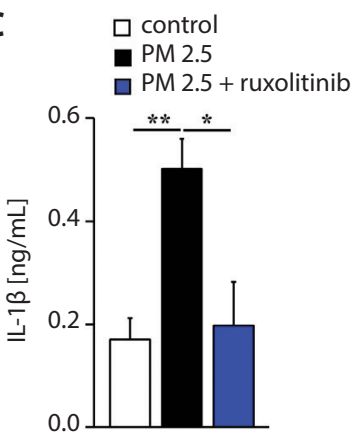

B
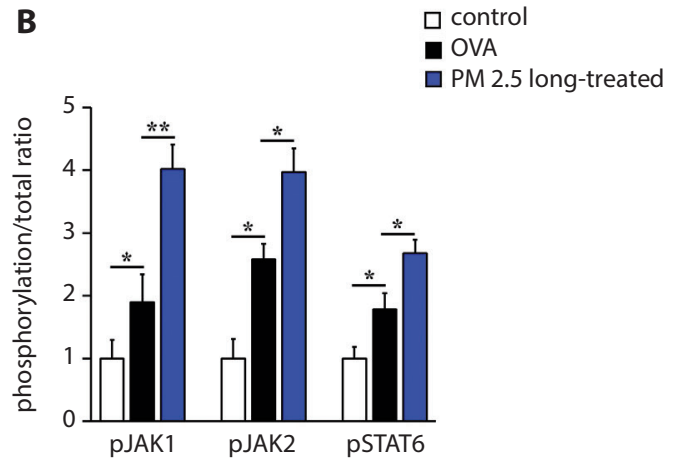

D
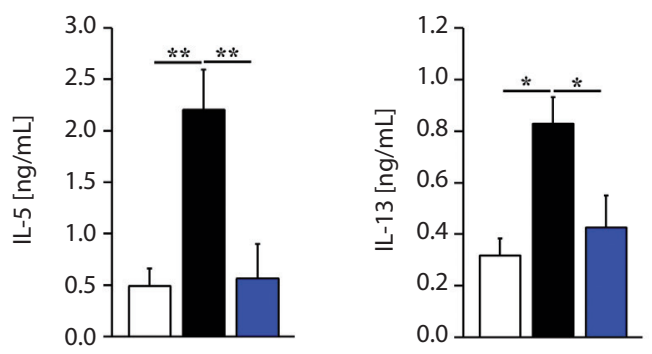

Fig. 5. JAK2-STAT6 pathway drives production of inflammatory cytokines and eosinophil infiltration. A. Representative western blot showing increase in phosphorylated JAK1, JAK2 and STAT6 after PM2.5 treatment as compared to control group and OVA group. B. Phosphorylation of JAK1, JAK2 and STAT6 as calculated from western blots in control group (white bars), OVA group (black bars) and OVA + PM2.5 long-treatment group (blue bars),

$\mathrm{n}=6$. Measurements of medium (C) IL-1 $\beta$, (D) IL-5 and (E) IL-13 in control (white bars), PM2.5-treated (black bars) and PM2.5 + ruxolitinib-treated (blue bars) bone marrowderived eosinophils, $n=4$

$\mathrm{p}^{*}<0.05, \mathrm{p}^{* *}<0.01$. to confirm that the JAK-STAT6 pathway was involved in PM2.5 and asthma, bone marrow derived eosinophil was treated with PM2.5 with or without JAK1/2 inhibitor, ruxolitinib. PM2.5 increased IL-1 $\beta$ (p $<0.01$, Fig. 5C), IL-5 (p $<0.01$, Fig. 5D) and IL-13 ( $<<0.05$, Fig. 5E) production in eosinophil medium; their production was blocked by ruxolitinib treatment ( $<<0.05$, Fig. 5C,D).

\section{Discussion}

PM2.5 pollution in developing countries such as China poses a serious public health problem, as exposure to ambient PM2.5 exacerbates allergic diseases. The occurrence of asthma and allergic diseases have been on the rise in both developed and industrializing countries due to exposure to a variety of environmental pollutants in the air. ${ }^{21,37-39}$ As such, the effects of PM2.5 have been gaining public attention. We investigated the effects of PM2.5 on eosinophil recruitment in OVA-induced asthma. PM2.5 increased the severity of OVA-induced asthma as indicated increasing in lung injury, alveolar intervals, capillary dilations, and inflammatory leukocytes infiltration. PM2.5 treatment with OVA significantly increased the presence of inflammatory cytokines in the BALF as compared to OVA alone, which increased the number of infiltrating eosinophils and macrophages. The infiltration of eosinophils was regulated through the JAK2 and STAT6 pathway, as increased phosphorylation of both these proteins was observed through western blotting.

The role of eosinophils has been examined in an acute murine PM2.5 OVA-induced asthma model..$^{40}$ In that acute model, mice were first sensitized to OVA, before being exposed to PM2.5 and then challenged with OVA. Under these conditions, the authors observed significant infiltration of eosinophils but not macrophages exposed to $10 \mu \mathrm{g}$ and $100 \mu \mathrm{g}$ of PM2.5 compared to control group after OVA challenge. In agreement with the acute model, our study also observed an increase in eosinophils after PM2.5 pretreatment. With continuous exposure to PM2.5 (through long treatment), eosinophil infiltration into the lungs was further increased. Although no increase in macrophages was observed in PM2.5 pre-treatment, similar to the acute study, long treatment of PM2.5 did significantly increase macrophage recruitment. As neutrophil recruitment during exposure to concentrated ultrafine airborne particles remains debated, ${ }^{40,41}$ neutrophil infiltration was not measured in our study.

During asthma, the presence of $\mathrm{T}_{\mathrm{H}} 2$ cytokines IL-4, IL-5 and IL-13 are typically observed during clinical manifestation of disease. ${ }^{42}$ However, particulate matter is known to induce the production of different cytokines due to the activation of diverse cellular and molecular mechanisms. For example, increased levels of IL-17 were observed, but there were no changes in IL-4. In another study, high levels of cytokines (IL-1 $\beta$ and IL-12), not typically associated with $\mathrm{T}_{\mathrm{H}} 2$ responses, were observed. ${ }^{34}$ This was in addition to increases in IL-5 and IL-13. Chemokines such as MCP-1 (which recruits monocytes and macrophages) were also detected. In another study, increases in IL-4 and IL-10 with decrease in interferon gamma (IFNY) were observed. ${ }^{40}$ In this study, PM2.5 long treatment increased the levels of IL-1 $\beta$, IL-12, IL-5, and IL-13. The increased level of IL-12 may have been induced by the macrophage 
recruitment in BALF (Fig. 3D). Alveolar macrophages are primed for the production of IL-10 and IL-12 in asthma. ${ }^{43}$ However, IL-12 could induce the type I immune response which is antagonistic with type II immune responses. Macrophages appear more as a consequence than as initiating factor of the $\mathrm{T}$-cell $\mathrm{T}_{\mathrm{H}} 2$ commitment and could be involved in the resolution of the asthmatic inflammation. Together with the chemokine MCP-1, these cytokines were important in the recruitment of macrophages and eosinophils into the lung.

The JAK-STAT pathway is important in the development of asthma. ${ }^{36}$ For example, IL-4 induces JAK1/ JAK3 resulting in STAT6 activation, which further polarizes the $\mathrm{T}_{\mathrm{H}} 2$ pathway. Another important pathway in asthma development is IL-13-JAK2-STAT6 pathway, which upon activation markedly increases mucous secretion. ${ }^{44}$ In this study, eosinophil recruitment was demonstrated to be reliant on the phosphorylation of JAK2 and STAT6. Ovalbumin treatment alone triggers this pathway but co-treatment with PM2.5 significantly increases the amount of phosphorylation of both JAK2 and STAT6. As a result of JAK2/STAT6 pathway activation, IL-13 was significantly increased and contributed to airway inflammation.

There are several limitations to our study. First, the polycyclic aromatic hydrocarbons, chemicals and metals were not analyzed in our study. Although these components are typically found in PM2.5, PM2.5 from different cities are composed of different compounds, which induces different responses in vivo. In addition, this meant that the component which contributed most to asthma exacerbation in PM2.5 long, continuous treatment could not be identified. Second, as the data is from a single city, it might be challenging to extend these findings to other cities due to differences in climate and pollution content. Finally, cytokines typically associated with asthma (such as IL-5 and IL-13), pro-inflammatory cytokines (IL-1 $\beta$ and IL-12) and leukocyte chemokines (MCP-1 and PGD 2 ) were observed to increase during PM2.5 long treatment in this study. These factors isolated from the BALF likely contributed to the induction of leukocyte recruitment into the lung and resulted in exacerbating the disease. However, the mechanistic contribution of individual cytokines to eosinophil recruitment was not examined in this study.

\section{Conclusions}

Although the effect of PM2.5 has been examined by other researchers, their studies have focused on the acute effects of PM2.5 on aggravating asthma. By providing continuous treatment of PM2.5 together with OVA treatment, our study demonstrates that prolonged exposure of PM2.5 increases the severity of asthma through exacerbated inflammation by increasing eosinophil recruitment.

\section{ORCID iDs}

Yonghui Yang (iD https://orcid.org/0000-0001-5955-2289 Xiaoxia Li (1) https://orcid.org/0000-0001-5401-2398 Xiaoying An (ID https://orcid.org/0000-0002-4799-8862 Ling Zhang (D) https://orcid.org/0000-0002-9313-4758 Xingbin Li (i) https://orcid.org/0000-0003-4411-1303 Liang Wang (D) https://orcid.org/0000-0002-5385-3045 Guiyun Zhu (D) https://orcid.org/0000-0002-9734-2001

\section{References}

1. Li L, Liu DJ. Study on an air quality evaluation model for Beijing City under haze-fog pollution based on new ambient air quality standards. Int J Environ Res Public Health. 2014;11(9):8909-8923.

2. Lu S, Wang Y, Teng Y, Yu X. Heavy metal pollution and ecological risk assessment of the paddy soils near a zinc-lead mining area in Hunan. Environ Monit Assess. 2015;187(10):627.

3. Xie J, Teng J, Fan Y, Xie R, Shen A. The short-term effects of air pollutants on hospitalizations for respiratory disease in Hefei, China. Int J Biometeorol. 2019;63(3):315-326.

4. Wang Y, Smith JA, Wilmers CC. Residential development alters behavior, movement, and energetics in an apex predator, the puma. PLoS One. 2017;12(10):e0184687.

5. Maki T, Puspitasari F, Hara K, et al. Variations in the structure of airborne bacterial communities in a downwind area during an Asian dust (Kosa) event. Sci Total Environ. 2014;488-489:75-84.

6. He M, Ichinose T, Song Y, et al. Effects of two Asian sand dusts transported from the dust source regions of Inner Mongolia and northeast China on murine lung eosinophilia. Toxicol Appl Pharmacol. 2013; 272(3):647-655.

7. Kim KH, Kabir E, Kabir S. A review on the human health impact of airborne particulate matter. Environ Int. 2015;74:136-143.

8. Cai Y, Shao Y, Wang C. The association of air pollution with the patients' visits to the department of respiratory diseases. J Clin Med Res. 2015;7(7):551-555.

9. de Haar C, Hassing I, Bol M, Bleumink R, Pieters R. Ultrafine but not fine particulate matter causes airway inflammation and allergic airway sensitization to co-administered antigen in mice. Clin Exp Allergy. 2006;36(11):1469-1479.

10. Singh S, Shi T, Duffin R, et al. Endocytosis, oxidative stress and IL-8 expression in human lung epithelial cells upon treatment with fine and ultrafine TiO2: Role of the specific surface area and of surface methylation of the particles. Toxicol Appl Pharmacol. 2007;222(2):141-151.

11. Primbs $T$, Simonich $S$, Schmedding D, et al. Atmospheric outflow of anthropogenic semivolatile organic compounds from East Asia in spring 2004. Environ Sci Technol. 2007;41(10):3551-3558.

12. Shadie AM, Herbert C, Kumar RK. Ambient particulate matter induces an exacerbation of airway inflammation in experimental asthma: Role of interleukin-33. Clin Exp Immunol. 2014;177(2):491-499.

13. Jacquemin B, Siroux V, Sanchez M, et al. Ambient air pollution and adult asthma incidence in six European cohorts (ESCAPE). Environ Health Perspect. 2015;123(6):613-621.

14. Baccarelli AA, Zheng Y, Zhang X, et al. Air pollution exposure and lung function in highly exposed subjects in Beijing, China: A repeatedmeasure study. Part Fibre Toxicol. 2014;11:51.

15. Hopke PK, Croft D, Zhang W, et al. Changes in the acute response of respiratory diseases to PM2.5 in New York State from 2005 to 2016. Sci Total Environ. 2019;677:328-339.

16. Hasunuma H, Yamazaki S, Tamura K, et al. Association between daily ambient air pollution and respiratory symptoms in children with asthma and healthy children in western Japan. J Asthma. 2018;55(7): 712-719.

17. Jo EJ, Lee WS, Jo HY, et al. Effects of particulate matter on respiratory disease and the impact of meteorological factors in Busan, Korea. Respir Med. 2017;124:79-87.

18. Mar TF, Koenig JQ, Primomo J. Associations between asthma emergency visits and particulate matter sources, including diesel emissions from stationary generators in Tacoma, Washington. Inhal Toxicol. 2010;22(6):445-448.

19. Vempilly J, Abejie B, Diep V, Gushiken M, Rawat M, Tyner TR. The synergetic effect of ambient PM2.5 exposure and rhinovirus infection in airway dysfunction in asthma: A pilot observational study from the Central Valley of California. Exp Lung Res. 2013;39(10):434-440. 
20. Iskandar A, Andersen ZJ, Bonnelykke K, Ellermann T, Andersen KK, Bisgaard $\mathrm{H}$. Coarse and fine particles but not ultrafine particles in urban air trigger hospital admission for asthma in children. Thorax. 2012;67(3):252-257.

21. Tecer LH, Alagha O, Karaca F, Tuncel G, Eldes N. Particulate matter (PM(2.5), PM(10-2.5), and PM(10)) and children's hospital admissions for asthma and respiratory diseases: A bidirectional case-crossover study. J Toxicol Environ Health A. 2008;71(8):512-520.

22. Yang CY, Tsai SS, Chang CC, Ho S-C. Effects of Asian dust storm events on daily admissions for asthma in Taipei, Taiwan. Inhal Toxicol. 2005; 17(14):817-821.

23. Yoo Y, Choung JT, Yu J, Kim KD, Koh YY. Acute effects of Asian dust events on respiratory symptoms and peak expiratory flow in children with mild asthma. J Korean Med Sci. 2008;23(1):66-71.

24. Watanabe M, Yamasaki A, Burioka N, et al. Correlation between Asian dust storms and worsening asthma in Western Japan. Allergol Int. 2011;60(3):267-275.

25. Kanatani KT, Ito I, Al-Delaimy WK, et al. Desert dust exposure is associated with increased risk of asthma hospitalization in children. Am J Respir Crit Care Med. 2010;182(12):1475-1481.

26. Dougherty RH, Fahy JV. Acute exacerbations of asthma: Epidemiology, biology and the exacerbation-prone phenotype. Clin Exp Allergy. 2009;39(2):193-202.

27. Kim HY, DeKruyff RH, Umetsu DT. The many paths to asthma: Phenotype shaped by innate and adaptive immunity. Nat Immunol. 2010; 11(7):577-584.

28. Miyauchi K. Helper T cell responses to respiratory viruses in the lung: Development, virus suppression, and pathogenesis. Viral Immunol. 2017;30(6):421-430.

29. Kirstein F,Nieuwenhuizen NE, Jayakumar J, HorsnellWGC, BrombacherF. Role of IL-4 receptor alpha-positive CD4(+) T cells in chronic airway hyperresponsiveness. J Allergy Clin Immunol. 2016;137(6):1852-1862.e9.

30. Horikawa K, Takatsu K. Interleukin-5 regulates genes involved in B-cell terminal maturation. Immunology. 2006;118(4):497-508.

31. McKenzie GJ, Bancroft A, Grencis RK, McKenzie AN. A distinct role for interleukin-13 in Th2-cell-mediated immune responses. Curr Biol. 1998;8(6):339-342.

32. Lumeng CN. Lung macrophage diversity and asthma. Ann Am Thorac Soc. 2016;13(Suppl 1):S31-S34.
33. Fricker M, Gibson PG. Macrophage dysfunction in the pathogenesis and treatment of asthma. Eur Respir J. 2017;50(3):1700196.

34. He M, Ichinose T, Yoshida Y, et al. Urban PM2.5 exacerbates allergic inflammation in the murine lung via a TLR2/TLR4/MyD88-signaling pathway. Sci Rep. 2017;7(1):11027.

35. Nials AT, Uddin S. Mouse models of allergic asthma: Acute and chronic allergen challenge. Dis Model Mech. 2008;1(4-5):213-220.

36. Pernis AB, Rothman PB. JAK-STAT signaling in asthma. J Clin Invest. 2002;109(10):1279-1283.

37. Li S, Batterman S, Wasilevich E, et al. Association of daily asthma emergency department visits and hospital admissions with ambient air pollutants among the pediatric Medicaid population in Detroit: Time-series and time-stratified case-crossover analyses with threshold effects. Environ Res. 2011;111(8):1137-1147.

38. Lerchl A. Comments on "Radiofrequency electromagnetic fields (UMTS, 1,950 MHz) induce genotoxic effects in vitro in human fibroblasts but not in lymphocytes" by Schwarz et al. (Int Arch Occup Environ Health 2008: doi:10.1007/s00420-008-0305-5). Int Arch Occup Environ Health. 2009;82(2):275-278.

39. Nordenhall C, Pourazar J, Ledin MC, Levin JO, Sandström T, Adelroth E. Diesel exhaust enhances airway responsiveness in asthmatic subjects. Eur Respir J. 2001;17(5):909-915.

40. Zhang X,Zhong W, Meng Q, etal. Ambient PM2.5 exposure exacerbates severity of allergic asthma in previously sensitized mice. J Asthma. 2015;52(8):785-794.

41. Kleinman MT, Sioutas C, Froines JR, et al. Inhalation of concentrated ambient particulate matter near a heavily trafficked road stimulates antigen-induced airway responses in mice. Inhal Toxicol. 2007; 19(Suppl 1):117-126.

42. Barnes PJ. Targeting cytokines to treat asthma and chronic obstructive pulmonary disease. Nat Rev Immunol. 2018;18(7):454-466.

43. Magnan $A$, van Pee $D$, Bongrand $P$, et al. Alveolar macrophage interleukin (IL)-10 and IL-12 production in atopic asthma. Allergy. 1998;53: 1092-1095.

44. Ding F, Liu B, Zou W, et al. LPS exposure in early life protects against mucus hypersecretion in ovalbumin-induced asthma by downregulation of the IL-13 and JAK-STAT6 pathways. Cell Physiol Biochem. 2018;46(3):1263-1274. 